\title{
A AVALIAÇÃO DOS FATORES DETERMINANTES PARA O AUMENTO DA PRODUTIVIDADE EM UMA EMPRESA PRODUTORA DE DENDÊ
}

\section{THE EVALUATION OF THE DETERMINANTS FACTORS FOR THE INCREASE OF THE PRODUCTION IN AN OIL PALM COMPANY}

Pedro Pinz Klemann ${ }^{1, *}$, Itallo Michael Soares Leal ${ }^{2}$, Claudionor Andrade Farias Junior ${ }^{3}$, Renato Martins Das Neves $^{3}$, André Augusto Azevedo Montenegro Duarte ${ }^{3}$

${ }^{1}$ Universidade da Amazônia, Centro de Estudos Sociais Aplicados, Ananindeua, Pará, Brasil

${ }^{2}$ Universidade Federal Rural da Amazônia, Instituto de Ciências Agrárias, Belém, Pará, Brasil

${ }^{3}$ Universidade Federal do Pará, Programa de Pós Graduação em Engenharia Civil, Belém, Pará, Brasil

${ }^{*}$ Corresponding author. Universidade da Amazônia, Ananindeua, Pará, Brazil Phone: +55 $9198410-0068$

e-mail addressl: pedroklemann@gmail.com (KLEMANN, Pedro Pinz).

\begin{tabular}{l} 
A R T I C L E I N F O \\
\hline Article history: \\
Received 2020-07-10 \\
Accepted 2020-09-04 \\
Available online 2020-09-04 \\
pal a vras - chave \\
Produtividade \\
Dendê \\
Palma de Óleo \\
Qualidade de Vida \\
Equipamentos \\
$k$ e y w ords \\
Productivity \\
Pailm Oil \\
Quality of life \\
Equipments
\end{tabular}

\begin{abstract}
A B S T R A C T
With the growth of the world population, we have increasingly sought to increase productivity in the agricultural sector, which increasingly requires techniques, tools and skilled labor to reach the satisfactory level of production. The objective of this article is to analyze the factors that may contribute to the increase of productivity in a company producing Dendê. Characterized as a descriptive exploratory research, where the data collection occurred with the application of a closed questionnaire, the main results demonstrate that the investigated company needs to invest in training of its supervisors and the quality of life of its employees so that there is the compliance with the activities and that they are executed through the best method, which allows the reduction of production costs. It is also worth noting that based on the analyzed data, it is necessary to invest constantly in the improvement of its machinery, in order to remain competitive before the market. Regarding the cultivation of the Oil Palm, the company applies strict fertilization and spacing standards among the seedlings, in addition to being geographically located in a region with a favorable climate for cultivation

R E S UM O

Com o crescimento da população mundial, tem-se buscado cada vez mais o aumento da produtividade no setor agrícola, que cada vez mais necessita de técnicas, ferramentas e mão de obra qualificada para se chegar no nível de produção satisfatório. O presente artigo tem como objetivo analisar os fatores que podem contribuir para a elevação da produtividade em uma empresa produtora de Dendê. Caracterizado como uma pesquisa exploratória descritiva, onde a coleta de dados se deu com a aplicação de um questionário fechado, os principais resultados demonstram que a empresa investigada necessita investir em capacitação de seus supervisores e na qualidade de vida dos seus funcionários de modo que haja o cumprimento das atividades e que as mesmas sejam executadas através do melhor método, que possibilite a redução dos custos de produção. Ressalta-se ainda que baseado nos dados analisados, fazse necessário investir constantemente na melhoria de seu maquinário, com o objetivo de se manter competitiva perante o mercado. No que tange ao cultivo da Palma de Óleo, a empresa aplica padrões rígidos de adubação e espaçamento entre as mudas, além de estar geograficamente localizada em uma região com clima favorável ao cultivo.
\end{abstract}




\section{INTRODUÇÃO}

O crescente número de habitantes em nosso planeta tem gerado discussões a respeito do aproveitamento do território que é utilizado. Este desafio tem se tornado cada vez mais difícil quando se fala em atender a demanda de toda a população mundial, que necessita cada vez mais de serviços e produtos de qualidade, que estimula as organizações a se adaptarem as exigências do mercado, pois com clientes cada vez mais exigentes, torna-se um desafio para as organizações desenvolver novos métodos de produção ou adotar sistemas de gestão que possibilitem a sua continuidade no mercado (OLIVEIRA, DUARTE e MONTEVETHI, 2002).

Pensando no mercado, as empresas buscam tornar-se competitivas como modo de sobrevivência, pois precisam atender as necessidades do mercado oferecendo produtos e serviços de qualidade a preços competitivos, de modo que consigam ter a rentabilidade desejada, para que possam investir em estratégias que a tornem um diferencial competitivo perante as demais

O avanço da tecnologia tem propiciado a introdução de novas técnicas e produtos para redução dos custos operacionais dentro das lavouras e das empresas. Uma dessas tecnologias é o Biodiesel, combustível que tem sua matéria prima extraída da Palma, cujo cultivo chama-se Dendeicultura. Essa é uma atividade ainda em expansão, mas que tem chamado a atenção para o Estado do Pará devido às condições climáticas favoráveis para o cultivo.

O objeto de estudo desta pesquisa é uma empresa produtora de Dendê, que atua no mercado desde 2009. Possui uma administração fechada devido ser de um país estrangeiro, investe constantemente em treinamento para a melhoria contínua de seus processos e consequentemente o aumento de sua produção buscando tornar-se competitiva, devido estar localizada no Município de Moju, no estado do Pará, um dos maiores polos produtores da Palma.

O presente trabalho tem como objetivo geral analisar os fatores que podem contribuir para a elevação da produtividade, de modo que seus custos de produção se mantenham ou diminuam.

A relevância desta pesquisa está no interesse pelo tema proposto, levando em consideração o estudo da produtividade. Das diversas atividades atreladas à administração da produção, este estudo visa avaliar somente as mais notáveis, que implicam diretamente na produção em questão.

\section{FUNDAMENTAÇ̃̃O TEÓRICA}

Em meados dos anos 1700, na Inglaterra, ocorreu uma grande quebra de paradigma que se chama Revolução Industrial, onde houve o advento das máquinas, inicialmente difundida com a substituição da forma humana e da água, pela força mecanizada e a instalação das unidades fabris.

Não se pode visualizar a Administração da Produção e Operações como um fator isolado, pois compete a uma relação direta ou indireta com diversos temas. As atividades que estão interligadas a gestão de produção apresentam indicadores elevados, pois ocorrem em uma proporção bem maior do que aparentam Reinaldo e Graeml (2007, p. 41).

De acordo com Martins e Laugeni (2006, p. 2) essas atividades são: Padronização dos produtos e seus processos de fabricação; o treinamento e habilitação da mão-de-obra direta; a criação e o desenvolvimento dos quadros gerenciais e de supervisão; o desenvolvimento de técnicas de planejamento e controle financeiro e da produção; e o desenvolvimento da técnica de vendas (MARTINS E LAUGENI, 2006, p. 2).

A relação entre o input, que são recursos, insumos, que implicam em valores ou quantidade, e o output que seria a medida quantitativa daquilo que foi produzido a partir do processo de transformação, pode estabelecer a quantificação da produtividade, que é um fator determinante no que diz respeito ao fracasso ou sucesso de uma empresa (MARTINS; LAUGENI, 2006)

O conceito de produtividade foi introduzido e desenvolvido como forma de auxiliar, avaliar e melhorar o desempenho das organizações. Por muito tempo a ideia de produtividade foi utilizada através da razão entre o resultado da produção e o número de empregados quando se almejava o aumento da produção por empregado. Além dessa, outras formas de medir a produtividade surgiram relacionando $o$ resultado da produção com outros recursos como a matériaprima, insumos, dentre outros (KING, 2007).

Leite et al (2016, p. 13) afirma que a produtividade é um dos focos gerenciais nas empresas. Isso porque o aumento contínuo da produtividade gera muitos benefícios seja para a própria organização ou até mesmo para a sociedade como um todo.

Produtividade segundo Corrêa e Corrêa (2010, p. 172), ñé uma medida de eficiência com que recursos de entrada (insumos) de um sistema de agregação de valor são transformados em saídas (produtos).

A melhoria constante do processo produtivo gera o aumento da qualidade, trabalho eficiente da mão-de-obra e equipamentos e consequentemente a redução dos custos de produção. Estes são fatores que podem impactar nos resultados finais do processo produtivo das organizações.

A importância de tornar o processo mais produtivo, e ter equipes de trabalho que conheçam de fato as atividades que desenvolvem e pelas quais são responsáveis, remete ao fato de que a falta de atenção ao manusear ou programar equipamentos, impacta negativamente a cadeia produtiva. Callado (2006, p. 23) afirma que ñ.. além de causarem problemas diretos à sua produção, influenciam no clima organizacional e representam custos diretos e indiretos...ò.

No Estado do Pará, a participação da agricultura familiar como mão-de-obra no cultivo do dendê, tem feito com que a integração da cultura do dendezeiro às demais atividades produtivas, tanto locais quanto regionais, seja considerada, buscando a produção de alimentos, para fomentar a mão-deobra utilizada nos dendezais, dando prioridade ao treinamento/capacitação de recursos humanos, entre outros (MÜLLER, 2006).

Em conformidade com Becker (2010), a cultura da palma de óleo necessita de uma extensa mão-de-obra braçal devido a colheita ser manual. $\mathrm{O}$ fato de não ser mecanizada tornase uma grande oportunidade para o desenvolvimento da agricultura familiar, que acabará contribuindo para a formação de pequenos 
produtores (BECKER, 2010).

A produção agrícola necessita de máquinas e implementos para que seja realizada, muito embora a agricultura necessite de mão-de-obra para execução de atividades relacionadas ao plantio, como a preparação do solo, adubamento, pulverização, colheita, dentre outras, as máquinas exercem um papel importantíssimo e auxiliam na produção, assim como as máquinas necessitam de operadores. Sobre isso, Slack et al (2009) ressaltam que nenhuma tecnologia opera totalmente sem a intervenção humana, pois, em alguma medida, todas necessitam dessa intervenção durante algum tempo.

Em entrevista dada para a Agroanalysis, cujo tema atribuído foi ñTecnologia significa produtividadeò, publicada em abril de 2011, Celso Cassale, Presidente da Câmara Setorial de Máquinas e Implementos Agrícolas (CSMIA) da Associação Brasileira da Indústria de Máquinas e Equipamentos (ABIMAQ), ressaltou a importância das máquinas e implementos para a produção agrícola, sendo o país considerado referência na produção de tais equipamentos, destacou que o objetivo desse investimento na produção de máquinas e implementos é voltado para o estímulo de uma agricultura autossustentável e que possibilite o desenvolvimento de produtores de diversos níveis. Cassale acrescentou ainda que ñAs máquinas e os implementos agrícolas deram competitividade para a agricultura brasileiraò.

A manutenção é um conjunto de ações indispensáveis ao funcionamento de máquinas, equipamentos, ferramentas e instalações, cuidados esses para que não haja a interrupção parcial ou total, causando assim, perda de produção além da elevação dos custos (MONTEIRO; SOUZA e ROSSI, 2010).

A busca pelo melhor aproveitamento de insumos, no que diz respeito a utilização de máquinas e equipamentos, reflete sobre a necessidade se realizar continuamente a manutenção preventiva, que compreende na inspeção feita pelos próprios trabalhadores, para que sejam evitadas interrupções na produção e as máquinas operem de acordo com o planejamento produtivo. Embora a manutenção preventiva tenha um custo mais elevado, é mais viável utilizada, pois com relação às quebras ocasionais, acaba saindo mais barato (MOREIRA, 2011).

A cultura do dendê cresceu expressivamente nos últimos anos, e chega à superar até mesmo a cultura da soja, no sentido de produção de óleo para a produção de bicombustível. A Associação Brasileira das Indústrias de Óleos Vegetais ï ABIOVE, através de relatório feito pelo economista Daniel Furlan Amaral, faz a seguinte afirmação: ñTendo em conta as restrições na oferta de óleo em cada mercado, a demanda mundial crescente por óleo para uso alimentar impulsionou, muito antes do surgimento da demanda da indústria de biodiesel, o crescimento da produção de oleaginosas com teor de óleo mais elevado que a soja. Ou seja, a demanda mundial por óleos vegetais incentivou o crescimento da produção de variedades cujo rendimento em óleo por hectare era mais vantajosoò (ABIOVE, 2009, p. 10)

No estado do Pará, os produtores de palma de óleo que possuem interesse em incentivar a pesquisa no sentido de buscar a criação de novas variedades de sementes, a partir do cruzamento das que já existem, contam com o apoio da Empresa Brasileira de Pesquisa Agropecuária ï EMBRAPA. Essas pesquisas são realizadas com a intenção de atender as necessidades do setor produtivo, a fim de combater doenças como o AF (Amarelecimento Fatal) que é a única com caráter totalmente irreversível, quando as mudas são atingidas pela mesma (MÜLLER, 2001).

Há diversas variedades de sementes, porém existem algumas que são mais produtivas com relação à área plantada (hectare). Existem algumas variedades de sementes que são de utilização comum entre os produtores de Palma de Óleo no Estado do Pará, dentre elas estão Deli x Ghana, Deli x Nigéria, Compacta x Ghana e Compacta x Nigéria. Vale ressaltar que pesquisadores utilizam nomenclaturas diferentes, porém na maioria dos casos trata-se da mesma variedade.

A expansão do cultivo do dendê é consequência da necessidade de atender as demandas globais por óleo vegetal, com uma acentuada procura em países desenvolvidos como a China e a Índia. No Brasil, apesar de ter a produção de óleo de soja mais elevada, tendo um percentual de 5,7 milhões de toneladas como percentual, fora o excedente que é exportado, o volume da produção de óleo de palma é uma crescente vertente neste cenário, segundo os dados publicados através do site da BiodiselBr, a dendeicultura cresceu expressivamente, tendo dado um salto de 95.000 para 250.000 toneladas por ano, quanto ao palmiste de 75.00 para 175.00 toneladas(BI ODISEL BR, 2013).

De acordo com o site, na região norte do país há o equivalente a 7 milhões hectares aptos ao plantio do dendê, sendo que só no Estado do Pará há uma representação dessa área no que diz respeito a 1,7 mil hectares, o que corresponde a $1,3 \%$ do território do Estado (BIODISEL BR, 2013).

A variabilidade de sementes nas mais diversas áreas da agricultura, permite que as empresas optem pelo melhor método de cultivo, quanto à escolha de variedades que sejam mais produtivas.

A figura abaixo apresenta um comparativo de todas as variedades de Palma de Óleo e suas principais características.

\begin{tabular}{|l|l|l|l|l|}
\hline VARIEDADE & $\begin{array}{l}\text { CRESCIMIENTO } \\
\text { DO TRONCO }\end{array}$ & AZEITE & FRUTO & AGLOMIERADO \\
\hline Deli X Gana & $55-60 \mathrm{~cm} /$ ano & $28-30 \%$ & $9-11 \mathrm{~g}$ & $22 \mathrm{~kg}$ \\
\hline Deli X Nigéria & $50-55 \mathrm{~cm} /$ ano & $28-30 \%$ & $9-11 \mathrm{~g}$ & $22 \mathrm{~kg}$ \\
\hline Compacta X Ghana & $40-45 \mathrm{~cm} /$ ano & $28-30 \%$ & $9-11 \mathrm{~g}$ & $18-22 \mathrm{~kg}$ \\
\hline Compacta X Nigéria & $40-45 \mathrm{~cm} /$ ano & $28-30 \%$ & $9-11 \mathrm{~g}$ & $18-22 \mathrm{~kg}$ \\
\hline
\end{tabular}

Figura 1 - Comparativo de variedades de Dendê (Fonte: ASD Costa Rica).

Vale ressaltar que há uma variedade de semente denominada híbrida, que é resultado do cruzamento da espécie americana Caiaué (Elaeis Oleifera) e o dendezeiro (E.guineenis Jacq.) de origem Africana. Esta espécie foi explorada com o Objetivo de desenvolver uma semente produtiva e resistente a pragas e doenças, principalmente a do amarelamento fatal (CHIA et al, 2009)

\section{METODOLOGIA}

Neste estudo, os objetivos estão relacionados à identificação dos fenômenos que influenciam no aumento da produtividade do objeto de estudo. Tratou-se de uma pesquisa básica, e com isso com base nos seus objetivos, é uma pesquisa 
exploratória descritiva, pois começou antes do planejamento do trabalho, e teve como Objetivo proporcionar as informações sobre o assunto que pesquisado, delimitando o tema, fixando as ideias dos objetivos e hipóteses (PRESTES, 2005).

A partir da classificação da pesquisa como sendo exploratória descritiva, que dá mais ênfase ao levantamento de teorias que abordem o tema em questão de maneira mais aprofundada, faz-se necessária também a classificação da pesquisa ainda de maneira conceitual, porém operativa. Desta maneira, com base nos procedimentos técnicos é um estudo de caso.

Sendo o Objetivo da pesquisa avaliar os fatores determinantes para o aumento da produtividade de uma empresa produtora de dendê, tratando-se de um estudo de caso, a amostra foi representada por $100 \%$ do universe (população), representado por 45 colaboradores atuantes no cultivo e manejo da Palma de Óleo.

Para a coleta de dados, realizou-se a aplicação de um questionário fechado com perguntas quantitativas, com linguagem acessível, em função da necessidade de adequação metodológica para o alcance do Objetivo deste estudo, onde buscou-se a coleta de informações para as questões relacionadas ao problema da pesquisa proposta.

O questionário buscou levantar as informações que fossem relevantes e pudessem contribuir para o atingimento do Objetivo deste estudo, com questões fechadas e objetivas de múltipla escolha, que foram aplicadas aos colaboradores atuantes no cultivo e manejo da Palma de Óleo.

A validação do questionário foi realizada após a aplicação de pré-teste em colaboradores aleatórios, tendo em vista a possibilidade de aprimoramento e validação. Depois de validado e sem alterações significativas, o questionário foi aplicado sem interferência dos entrevistados.

A tabulação dos dados coletados foi feita através da organização em tabelas, onde as informações foram classificadas e reunidas de forma que as hipóteses pudessem ser comprovadas ou refutadas.

A organização dos dados foi realizada de modo que se permitiu fazer uma análise estatística que foi apresentada através de gráficos, de tal forma que, as informações coletadas pudessem ser mais bem compreendidas e interpretadas rapidamente.

A avaliação dos resultados se deu com o agrupamento dos dados necessários para que pudessem ser determinados os indicadores que facilitaram a análise dos dados obtidos. A partir da análise do material coletado, elaborou-se um relatório estatístico de respostas por questões, que objetivou dar subsídio à solução do problema de pesquisa proposto.

\section{RESULTADOS OBTIDOS}

Por meio deste trabalho tornou-se possível verificar a realidade da empresa quanto às atividades pertinentes aos fatores determinantes para a avaliação da produtividade, de modo que ascendesse uma gama de conclusões relevantes bem como se dá a concepção dos colaboradores diante dos mesmos.

As evidências levantadas deixaram claro o perfil da amostra da presente pesquisa, por entender que os fatores gênero, faixa etária e escolaridade têm impacto direto na condução dos trabalhos no cultivo do Dendê. Atualmente, é uma organização onde há predominantemente pessoas do sexo masculino com idades entre 25 a 35 anos, com o $1^{\circ}$ grau incompleto.

No que se refere à compreensão e à obtenção da elevação da produtividade pelos funcionários do cultivo do Dendê, de forma que se torne possível a efetivação dos resultados obtidos através da avaliação da mão-de-obra, equipamentos/implementos e variedade de sementes, vale ressaltar as principais considerações:

Foi percebido que o fato da instrução ser baixa, estes tiveram direta relação na concepção dos funcionários quanto ao significado de produtividade, os fatores que influenciam no seu aumento, além de opiniões pessoais de cada um sobre a condução dos trabalhos pelos próprios supervisores de campo, o uso de ferramentas e até o conhecimento sobre as diferentes variedades que a empresa cultiva.

Segundo a colocação de Callado (2006), é sabido que a mão-de-obra qualificada é mais produtiva aliada a uma eficiente gestão de pessoas, de modo que os colaboradores não ajam de maneira mais improvisada e sim com mais profissionalismo. A qualificação da mão-de-obra foi o fator escolhido como sendo determinante para a elevação da produtividade pela maior parte dos funcionários $(62,22 \%)$, seguido pela Qualidade de Vida $(24,44 \%)$, Postura Gerencial $(11,12 \%)$ e Ferramentas $(2,22 \%)$.

Embora quase metade dos funcionários (48,89\%) afirmarem que são treinados mensalmente, uma parcela considerável $(37,77 \%)$ marcou a alternativa ñoutrosò, o que implica que não são treinados periodicamente.

É sabido que o objeto de estudo deste trabalho cultiva mais de 1 variedade de Palma de Óleo, onde cada variedade possui características que requerem um tratamento diferenciado para a obtenção da produtividade máxima. Segundo a opinião de mais da metade dos funcionários $(57,78 \%)$, frente ao conhecimento que possuem com relação à variedade de sementes, confirmam que a empresa planta as variedades de acordo com as especificações técnicas. Uma porcentagem relativamente alta $(15,55 \%)$ desconhece a respeito. Pode-se considerar este percentual como ponto positivo para a empresa quanto à elevação da produtividade.

Levando em consideração os dados coletados através da ASD ï Costa Rica (2012), que expõem as variedades de sementes e o espaçamento utilizado para cada uma delas, cuja capacidade produtiva por hectare varia de 143 a 170 mudas de palma de óleo, tendo em vista que a empresa utiliza um espaçamento correto entre as mudas no sentido de seguir as especificações técnicas condizentes com o plantio da Palma de óleo. Em contrapartida existe o fato dos investigados $(66,67 \%)$ alegarem que compreendem ser mais importante para a elevação da produtividade a adubação.

Com relação às variedades de Palma, mais de 50\% afirmaram que desconhecem sobre as variedades mais produtivas e grande parte dos colaboradores afirmam que a empresa respeita o padrão de espaçamento de cada variedade, além de considerar que a empresa adota um padrão de adubação rígido. Este fato torna-se incoerente, pois se eles desconhecem sobre as variedades, não são capazes de opinar sobre a adubação 
e o espaçamento necessário para cada variedade.

Considerando a importância da manutenção no sentido da busca pelo aumento da produtividade, quanto a não interrupção das atividades, visando tornar o processo produtivo mais rápido, de modo que se produza aquilo que o mercado demanda, os funcionários $(51,12 \%)$ caracterizam a aplicação da manutenção preventiva e corretiva como sendo frequente na empresa.

Foi levantado que $60 \%$ dos respondentes classificam pouco modernos os equipamentos e implementos que a empresa utilizada para a execução das atividades. O maquinário que apresenta baixo grau de modernidade é admitido como um fator que pode gerar ineficiência dentro da posição em que o mesmo se encontra dentro da cadeia produtiva, e isso remete a desperdício, o que pode acarretar custos desnecessários.

No que se refere à validação dos fatores avaliados como sendo classificados determinantes para a elevação da produtividade na empresa investigada, mais da metade $(51,11 \%)$ alega que a empesa disponibiliza recursos (máquinas, funcionário, insumos agrícolas) que contribuem para o aumento da produtividade, de maneira que haja o mantimento ou a redução dos custos operacionais.

Nota-se que há uma substancial satisfação dos funcionários quanto à qualidade de vida que a empresa disponibiliza $(71,11 \%)$ e que $51 \%$ afirmam que a empresa dá condições de vida, dentre outros, para o aumento da produtividade.

Quanto ao clima da região de Moju/PA, onde a empresa possui suas instalações pertinentes ao cultivo do dendê, a maioria dos funcionários $(93,33 \%)$ afirma ser muito favorável para tal segmentação. Isso pode ser constatado através das colocações de Marzullo (2007), que salienta a necessidade que este genótipo tem quanto ao clima, no sentido de se desenvolver em climas quentes e úmidos como o da referida região.

\section{CONCLUSÃO}

Frisar a relevância de uma avaliação dos fatores que podem ser geradores de custos, e que refletem na baixa produtividade, torna-se indispensável hoje em dia. Considerando o cenário em que as empresas estão inseridas, que está cada vez mais competitivo, isso exige das organizações uma postura que tende para esse viés de dar uma atenção especial aos recursos utilizados de modo que a gestão dos mesmos seja voltada para a elevação da produtividade.

A aplicação de novas tecnologias tem proporcionado o aumento constante no rendimento das lavouras e tem feito do Brasil destaque no mundo com a necessidade crescente por alimentos. Para as empresas fornecedoras de serviços e aos grandes produtores, tornar-se o mais eficiente possível, utilizando o mínimo de recursos buscando a maximização de seus rendimentos com as atividades que realizam, tem se tornado uma busca constante pois o agronegócio tem se tornado uma das atividades mais importantes para o desenvolvimento econômico do Brasil.

Destaca-se que no Estado do Pará, a agricultura familiar vem recebendo uma atenção especial, no que diz respeito à utilização da mão-de-obra no cultivo do Dendê, realizando a intercepção da cultura do dendeizeiro com outras atividades que são consideradas mais produtivas, dando prioridade ao treinamento/capacitação de recursos humanos, afim de fomentar a mão-de-obra adotada nos dendezais.

Por fim, pode-se concluir que quando se trata do aumento da produtividade, deve-se ter a plena convicção de que isso requer mudanças e quebras de paradigma de qualidade, tecnologia e na organização do trabalho, de forma que esta trata de benefícios à organização com o aumento da satisfação dos clientes, redução dos desperdícios e melhor utilização dos recursos humanos.

\section{REFERÊNCIAS}

ALMEIDA, D. P. Racionalização industrial. 2003 (MIMEO).

AMARAL, Daniel Furlan. Desmistificando o Programa Nacional de Produção e uso do Biodiesel: A visão da indústria Brasileira de óleos vegetais. São Paulo: Associação Brasileira das Indústrias de Óleos Vegetais, 2009.

ASD Costa Rica. Deli X Ghana (Variedade de alta densidade). ASD Costa Rica. Disponível em:<http://www.asdcr.com/index.php. Acesso em : 07 set. 2019.

ASD Costa Rica. Compacta X Ghana(Variedade de alta densidade). ASD Costa Rica. Disponível em:

<http://www.asd-cr.com/index.php. Acesso em: 07 set. 2019.

ASD Costa Rica. Compacta X Nigéria. ASD Costa Rica. Disponível em

<http://www.asdcr.com/index.php.Acesso em : 07 set. 2019.

BECKER, K. Bertha. Recuperação de áreas desflorestadas da Amazônia: será pertinente o cultivo da palma de óleo (Dendê)?. Cofins [Online], 10 2010, posto online em 14 janvier 2011, consultado o 20 juillet 2011. URL: http://cofins.revues.org/6609; DOI: 10.4000/confins.6609

CALlADO, Antonio André Cunha. Agronegócio. 1. ed. São Paulo: Atlas, 2006.

CASALE, Celso. Tecnologia significa produtividade, abr. 2011. Disponível em: <http://bibliotecadigital.fgv.br/ojs/index.php/agroanalysis /article/viewFile/25596/24452>. Acesso em: 09 set. 2019.

CHIA et al.Repetibilidade de produção de cachos de híbridos interespecíficos entre o Caiaué e o dendezeiro. v. 39, p. 249-254. 2009.

CHIAVENATO, Idalberto. Administração nos novos tempos. 2. ed. Rio de Janeiro: Elsevier, 2010.

FRAGA, Alana; BORIN, Filipe. A evolução dos óleos vegetais, março 2013. Disponível em: http://www.biodieselbr.com/noticias/usinas/insumo/aevolucao-oleos-vegetais-040313.htm. Acesso em: $30 \mathrm{de}$ abril de 2019.

CORRÊA, Henrique L; CORRÊA, Carlos A. Administração da produção e operações: manufatura e serviços: uma abordagem sistêmica. 2. ed. 5. reimpr. São Paulo; Atlas, 2010. 
KING, Ney Cesar de Oliveira. Desenvolvimento de um processo para análise da Produtividade Sistêmica. 2007. 167 f. Dissertação (Mestrado em Engenharia de Produção e Sistemas) -Pós Graduação em Engenharia de Produção e Sistemas, Pontifica Universidade Católica do Paraná, Curitiba, 2007.

LEITE, Jussara Fernandes. et al. Análise do Layout do processo de fabricação de tijolos: Um estudo de caso para melhoria da produtividade e da segurança e saúde dos funcionários. In: XXXVI ENCONTRO NACIONAL DE ENGENHARIA DE PRODUÇÃO. 2016, João Pessoa. Anais: João Pessoa: ENEGEP, 2016. P. 13-29.

MARTINS, Petrônio Garcia, LAUGENI, Fernando Piero. Administração da Produção. Saraiva, São Paulo, 2006. 2 ed. 9 - 11 p.

MARZULLO, R.C.M. Análise da Ecoeficiência dos Óleos Vegetais Oriundos da Soja e da Palma, Visando a Produção de Biodiesel. Dissertação de M.Sc., Escola Politécnica, USP, São Paulo, SP, Brasil, 2007.

MONTEIRO, Caio Italiano; SOUZA, Leandro Ramalho de; ROSSI, Paulo Henrique Lobo. Manutenção Corretiva: Manutenção e Lubrificação de Equipamentos, set. 2010. Disponível em: http://wwwp.feb.unesp.br/jcandido/manutencao/Grupo_6. pdf>. Acesso em: 07 nov. 2019

MOREIRA, Daniel Augusto. Administração da produção e operações. São Paulo, Pioneira, 1993. 600 p.

MOREIRA, Daniel Augusto. Administração da produção e operações. 2. ed. rev. e ampl. São Paulo: Cengage Learning, 2011.
MOREIRA, Daniel Augusto. Introdução à administração da produção e operação. São Paulo: Pioneira,1998.

MULLER, Antonio Agostinho et al. Agronegócio do Dendê. 1. ed. Belém: Embrapa Amazônia Ocidental, 2001.

OLIVEIRA, F. A.; DUARTE, R. N.; MONTEVECHI, J. A. B. $O$ reflexo da mudança organizacional sobre o desempenho de uma empresa de autopeças: um estudo de caso. In: XXII Encontro Nacional de Engenharia de Produção, 2002, Curitiba. CD - room XXII ENEGEP, 2002.

REINALDO, Jurandir; GRAEML, Alexandre R. Administração da produção: operações industriais e de serviços. Curitiba: UnicenP, 2007, $41 \mathrm{p}$.

PRESTES, Maria Luci de Mesquita. A Pesquisa e a Construção do Conhecimento Científico: Do planejamento aos textos, da escola a academia. 3. ed. São Paulo: Rêspel, 2005. 26 p.

SLACK, N. et al. Administração da Produção. 3. ed. São Paulo: Atlas, 2009.

STONER, James A. F et al. Administração. 5. ed. Rio de Janeiro: Livros Técnicos e Científicos Editora S.A, 1999. $46 \mathrm{p}$.

UNITED STATES DEPARTAMENT OF AGRIGULTURE. Growing palm oil supplies temper global vegetable oil prices, December 2012. Disponível em: http://www.ers.usda.gov/data-products/chart gallery/detail.aspx ?chart $I d=33952 \&$ ref $=$ collection. Acesso em: 30 setembro de 2019. 